Check for updates

Cite this: RSC Adv., 2018, 8, 25526

Received 6th June 2018

Accepted 10th July 2018

DOI: $10.1039 / c 8 r a 04830 d$

rsc.li/rsc-advances

\section{Photoluminescence color stability of green- emitting InP/ZnS core/shell quantum dots embedded in silica prepared via hydrophobic routes $\dagger$}

\author{
Taichi Watanabe, ${ }^{a}$ Yoshiki Iso, (D) *a Tetsuhiko Isobe (D) *a and Hirokazu Sasaki ${ }^{b}$
}

In this work, green-emitting InP/ZnS quantum dots (QDs) modified with 1-dodecanethiol were embedded into silica by two methods to improve their photostability while maintaining a high photoluminescence quantum yield (PLQY) and a color coordinate. A monolithic QD-silica composite prepared by a nonaqueous route with tetraethyl orthosilicate and lactic acid featured low transparency, a loss of the color purity of green, and a PLQY of $1.6 \%$, which was considerably lower than that of the original QDs (67\%). The decrease of the PLQY was attributed to QD aggregation in the sol-gel process and degradation of the QDs by the acid. The alternative method involved stirring a toluene dispersion of the QDs with tetramethyl orthosilicate (TMOS) for $20 \mathrm{~h}$ or 7 days. The PLQY of the TMOS-modified InP/ZnS QDs (20 h) was $62 \%$, which was only slightly lower than that of the original QDs. The PLQY decreased to $52 \%$ when the duration of aging was prolonged to 7 days. This decrease was attributed to desorption of surface modifiers from the QD surface and oxidative degradation by oxygen dissolved in toluene. Herein, the color coordinate was maintained stably. Photostability was evaluated by continuous irradiation of the samples by a blue light emitting diode. The decrease of photoluminescence $(P L)$ intensity was suppressed by the silica encapsulation. In particular, the PL intensity of the TMOS-modified InP/ZnS QD sample (7 d) maintained $99 \%$ of its initial intensity. Silica encapsulation of InP/ZnS QDs prevented contact of the QDs with oxygen in the air, resulting in improved photostability.

\section{Introduction}

Fluorescent quantum dots (QDs) are nano-sized crystals of a semiconductor, which have widened bandgaps owing to quantum size effects. QDs are useful for a range of applications such as displays, white light emitting diodes (LEDs), and bioimaging owing to their tunable emission wavelength and high photoluminescence quantum yields (PLQYs). Although CdS and CdSe QDs are representative materials showing strong light emission in the visible spectrum, the high toxicity of $\mathrm{Cd}$ is

${ }^{a}$ Department of Applied Chemistry, Faculty of Science and Technology, Keio University, 3-14-1 Hiyoshi, Kohoku-ku, Yokohama 223-8522, Japan. E-mail: iso@applc.keio.ac.jp; isobe@applc.keio.ac.jp; Fax: 8145566 1551; Tel: +81 45566 1558; +81 455661554 ${ }^{b}$ Shoei Chemical Inc., 2-1-1 Nishi-Shinjuku, Shinjuku-ku, Tokyo 163-0443, Japan $\dagger$ Electronic supplementary information (ESI) available: Photograph showing blue light irradiation of a sample (Fig. S1); photographs of TEOS dispersion of InP/ZnS QDs under white light and $365 \mathrm{~nm}$ near-UV light (Fig. S2); photographs of mixtures of TEOS and lactic acid after gelation (Fig. S3); detailed values of plotted color coordinates (Table S1); photographs of toluene dispersions of as-received InP/ZnS QDs and TMOS-modified InP/ZnS QDs ( $20 \mathrm{~h}$ and $7 \mathrm{~d}$ ) under white light and $365 \mathrm{~nm}$ UV light (Fig. S4); PLQYs of toluene dispersions of as-received InP/ZnS QDs without TMOS for the control experiments (Table S2); changes in PL spectra under continuous irradiation by blue LED (Fig. S5). See DOI: $10.1039 / \mathrm{c} 8 \mathrm{ra} 04830 \mathrm{~d}$ a serious obstacle to practical applications of these materials. Cd-free alternatives are therefore highly desired. InP QDs have attracted attention as a Cd-free material for applications to displays and bioimaging because of their efficient visible luminescence through a direct transition. ${ }^{1-4}$ This material is synthesized by non-aqueous liquid phase methods. ${ }^{5,6}$ Furthermore, core-shell type InP/ZnS QDs can be prepared by growth of a $\mathrm{ZnS}$ shell on the InP core to passivate surface defects that contribute to non-radiative relaxation. Yang et al. reported a PLQY of more than $60 \%$ for InP/ZnS QDs with a narrow emission peak, the position of which could be tuned in the visible region. ${ }^{6}$ Such fluorescent materials with high PLQYs and color purity are required for wide color gamut displays.

For applications of QDs to opto-electronic devices, the PL intensity of QDs should be maintained under conditions of long-term continuous excitation. However, InP QDs degrade through photooxidation of their surfaces. ${ }^{7}$ This problem is not completely resolved by $\mathrm{ZnS}$ shell growth over the InP core. ${ }^{6}$ Additional approaches to stabilizing QDs involve preventing contact with air by embedding the QDs in a transparent matrix, such as poly(methyl methacrylate) (PMMA) ${ }^{7,8}$ or silica. ${ }^{9}$ This encapsulation effectively suppresses photooxidation of the QD surface by oxygen. The gas-barrier properties and chemical 
stability of silica are superior to those of PMMA $;^{7}$ thus, protection of InP/ZnS QDs by silica shows great potential for improving their photostability.

Most composites of nanocrystals and silica are prepared by sol-gel methods involving alkoxysilanes. ${ }^{\mathbf{1 0 - 1 5}}$ One problem with these approaches is that $\mathrm{HCl}$ is generally used as a catalyst to promote gelation in the sol-gel technique; however, QDs are damaged by strong acid. Moreover, it is technically difficult to maintain the dispersion of QDs and avoid aggregation in the sol, which is a mixture of a hydrophilic liquid, lipophilic liquid, and an amphiphilic liquid with the desired composition. The aggregation of QDs increases the probability of self-absorption and is accompanied by a redshift of the emission peak and a decrease of the PLQY.

We have previously focused on a tetramethylammonium silicate (TMAS) aqueous solution as a silica source. ${ }^{16}$ Monolithic silica samples were fabricated from this solution through a solgel method following the addition of an ester compound as a gelling agent. ${ }^{17}$ In our previous work, we prepared monolithic and transparent TMAS-derived silica composites embedded with negatively-charged fluorescent nanoparticles, e.g., $\mathrm{YVO}_{4}: \mathrm{Bi}^{3+}, \mathrm{Eu}^{3+}{ }^{18} \mathrm{CuInS}_{2} / \mathrm{ZnS}$ QDs, ${ }^{19}$ and InP/ZnS QDs. ${ }^{16}$ In these works, hydrophilization of the QDs was required because they featured a hydrophobic surface owing to their modification with 1-dodecanethiol (DDT). Hydrophilization of the nanoparticles was achieved by exploiting interactions between amphiphilic molecules and hydrophobic ligands on the QD surface, ${ }^{\mathbf{2 0 , 2 1}}$ and ligand exchange of the hydrophobic ligands with hydrophilic ligands., ${ }^{9,22,23}$ We hydrophilized the $\mathrm{CuInS}_{2} / \mathrm{ZnS}$ and InP/ZnS QDs by exchanging adsorbed DDT with 3-mercaptopropionic acid (MPA), followed by dispersion in an aqueous TMAS solution and gelation. The fabricated silica composite with the embedded fluorescent nanoparticles was highly transparent and photostable under excitation. However, the PLQY of the InP/ZnS QDs decreased from $51.6 \%$ to $19.2 \%$ through the hydrophilization process. ${ }^{16}$ This decrease might be attributed to desorption of the surface ligands accompanied by the generation of surface defects. The reduced PLQY is a considerable disadvantage of the hydrophilization process by ligand exchange, as has been previously reported. ${ }^{22,24-28}$ Tamang et al. reported quenching of stearate-modified $\mathrm{InP} / \mathrm{ZnS}$ through hydrophilization by ligand exchange using various thiol molecules having a carboxylate group in their structure, which led to a $4-42 \%$ retention of the initial PL intensity. ${ }^{22}$ Hence, nonaqueous techniques for embedding hydrophobic QDs in a silica matrix without ligand exchange treatments are therefore required to suppress the PLQY decrease.

In this work, we report: (i) a non-aqueous fabrication of a monolithic silica composite containing green-emitting InP/ ZnS QDs via a sol-gel method and (ii) encapsulation of QDs into silica through modification of alkoxylsilane on the QD surface with only traces of water in a solvent. Method (i) involves the preparation of silica from carboxylic acid and an alkoxylsilane, as reported by Sharp. ${ }^{29}$ We fabricated monolithic silica composites through gelation of a mixture of a carboxylic acid and tetraethyl orthosilicate (TEOS) dispersion containing DDTmodified InP/ZnS QDs. A carboxylic acid with a $\mathrm{p} K_{\mathrm{a}}<4$ was required for gelation in this method. ${ }^{16}$ However, a strong acid would degrade the QDs. Therefore, lactic acid with a $\mathrm{p} K_{\mathrm{a}}=3.86$ was chosen. Method (ii) refers to an approach previously used by Huang et al. to improve the stability of QDs by silica encapsulation. ${ }^{30}$ They prepared silica spheres embedded with oleatemodified $\mathrm{CH}_{3} \mathrm{NH}_{3} \mathrm{PbBr}_{3}$ QDs by the addition of tetramethyl orthosilicate (TMOS) into a toluene dispersion of the QDs, through stirring at room temperature. The mechanism of silica generation was considered to be hydrolysis and condensation of TMOS on the QD surface owing to traces of water in the toluene solution $(0.0623 \%)$. Herein, TMOS was used because of its high hydrolyzation rate. The positive charge of silicon increases when the number of carbon atoms in the alkyl chain decreases. ${ }^{31}$ This results in the increase of the sensitivity toward hydrolysis; hence, TMOS is preferable to TEOS for this purpose. Silica composites embedded with InP/ZnS QDs can be fabricated by exploiting this acid-free method based on traces of water in the solvent. We characterized properties of our silica composites prepared by the above two methods including the structure, morphology, light absorption, color coordinate calculated from PL spectrum, PLQY, and photostability.

\section{Experimental section}

\subsection{Reagents}

A 1-octadecene dispersion of as-received InP/ZnS QDs capped with DDT (0.6 wt\%, SHOEI CHEMICAL), hexane (95.0\%, Kanto Chemical), toluene $(>99.5 \%$, Kanto Chemical; water content $\leq$ $0.03 \%)$, acetone $(>95.0 \%$, Taisei Chemical), lactic acid (85.092.0\%, Kanto Chemical), TEOS (99.9\%, Kanto Chemical), and TMOS (>99.0\%, Tokyo Chemical Industry) were used. Hexane, toluene, acetone, and TMOS were dehydrated over molecular sieves (3A 1/8, Wako Pure Chemical Industries) prior to use.

To prepare a dried powder of the as-received InP/ZnS QDs, dehydrated acetone $(20 \mathrm{~mL})$ was added to $6.0 \mathrm{~mL}$ of the QD dispersion, followed by ultrasonication. Precipitated QDs were obtained by centrifugation at $\sim 16000 \times g(12000 \mathrm{rpm}$, with a $10 \mathrm{~cm}$ diameter rotor) for $15 \mathrm{~min}$. After removal of the supernatant, the obtained precipitate was dispersed in a mixture of dehydrated toluene $(10 \mathrm{~mL})$ and dehydrated acetone $(20 \mathrm{~mL})$ under ultrasonication and centrifuged at $\sim 16000 \times g$ for $15 \mathrm{~min}$. This procedure was repeated twice. The powder sample was prepared by drying the final precipitate under vacuum for 1 day.

\subsection{Preparation of monolithic QD-silica composite by a sol- gel method with TEOS}

InP/ZnS QD powder (14 mg) was dispersed in TEOS $(1.5 \mathrm{~mL})$ by ultrasonication. The prepared TEOS dispersion of QDs $(9.3 \mathrm{mg}$ $\mathrm{mL}^{-1}$ ) was mixed with lactic acid $(1 \mathrm{~mL})$ under stirring for $2 \mathrm{~min}$ and then poured into a polystyrene mold $(25 \mathrm{~mm} \times 25 \mathrm{~mm} \times$ $15 \mathrm{~mm}$ ) before gelation. After drying in an electric desiccator for 5 days, a monolithic InP/ZnS QD-embedded silica composite sample was obtained. A certain degree of shrinkage was observed during drying. The final QD concentration was $1.1 \mathrm{wt} \%$, as calculated from the weight of the fabricated 
composite (1.28 g). The blank sample without QDs was also prepared from only TEOS and lactic acid in the same manner.

\subsection{Preparation of QD-silica composite by TMOS modification}

The as-received InP/ZnS QD powder $(12.8 \mathrm{mg})$ was dispersed in toluene $(20 \mathrm{~mL})$ by ultrasonication. Herein, as-purchased toluene was used without dehydration because a trace amount of water is needed for hydrolysis of the TMOS. The TMOS (100 $\mu \mathrm{L})$ was added to the QD dispersion $\left(0.64 \mathrm{mg} \mathrm{mL}^{-1}\right)$ and this dispersion was aged for $20 \mathrm{~h}$ or 7 days with stirring at room temperature. The aged dispersion was transferred to a roundbottom glass flask and then evaporated under reduced pressure at $30{ }^{\circ} \mathrm{C}$ for $90 \mathrm{~min}$ to remove toluene and residual TMOS. The remaining paste was dispersed in toluene $(1 \mathrm{~mL})$ for retrieval from the flask. This dispersion was finally vacuum dried for 1 day in a glass vial to yield TMOS-modified InP/ZnS QDs $(20 \mathrm{~h}$ or $7 \mathrm{~d}$ ). Dispersions of TMOS-modified InP/ZnS QDs (20 h and $7 \mathrm{~d}$ ) were also prepared by dispersing the dried QDs in dehydrated toluene.

\subsection{Characterization}

X-ray diffraction (XRD) profiles of dried samples were obtained with an X-ray diffractometer (Rint-2200, Rigaku) with a $\mathrm{Cu} \mathrm{K} \alpha$ radiation source and a monochromator. Elemental compositions of dried samples were determined by the fundamental parameter method using an X-ray fluorescence (XRF) analyzer (ZSX mini II, Rigaku). Fourier transform infrared (FT-IR) spectra of dried samples dispersed in $\mathrm{KBr}$ disks were measured on a spectrometer (FT/IR-4200, JASCO). The QD morphologies were observed with a field-emission transmission electron microscope (TEM; Tecnai 12, FEI). TEM samples were prepared by drying a drop of the QD dispersion on a collodion-coated copper TEM grid (COL-C10, Oken Shoji) in an electric desiccator for 1 day or more.
Ultraviolet/visible (UV-vis) absorption spectra of QD dispersions and transmission spectra of monolithic samples were measured with a UV/visible/near-infrared optical absorption spectrometer (V-570, JASCO). PL spectra were collected with a fluorescence spectrometer (FP-6500, JASCO). Color coordinates were calculated from the recorded PL spectra. The absolute PLQY values of the dried samples and monolithic composites were measured on the same spectrometer equipped with an integrating sphere (ISF-513, JASCO) based on the following equation:

$$
\Phi=\frac{I_{\mathrm{em}}}{I_{\mathrm{ex}}-I_{\mathrm{ref}}}
$$

where $\Phi$ is PLQY, $I_{\mathrm{em}}$ is the integrated emission intensity of the sample, $I_{\mathrm{ex}}$ is the integrated intensity of the incident excitation light $\left(\lambda_{\text {ex }}=468.3 \mathrm{~nm}\right)$, and $I_{\text {ref }}$ is the integrated intensity of the excitation light reflected by the sample. A reflectance standard (Spectralon SRS-99, Labsphere) was used to determine $I_{\mathrm{ex}}$. The absolute PLQYs of the QD dispersions at $\lambda_{\mathrm{ex}}=468.3 \mathrm{~nm}$ were measured with a quantum efficiency measurement system (QE2000, Otsuka Electronics). A flat panel blue LED (TE-4556, Nissin Electronics) equipped with a power supply (LPR-10W, Nissin Electronics) was used for photostability testing (see Fig. S1 in $\mathrm{ESI} \dagger$ ). Its luminescent wavelength and irradiance were $468.3 \mathrm{~nm}$ and $34 \mathrm{~W} \mathrm{~m}^{-2}$, respectively.

\section{Results and discussion}

\subsection{Characterization of monolithic InP/ZnS QD-silica composite}

Fig. 1 shows photographs of the monolithic blank silica without QDs and a silica composite containing InP/ZnS QDs. The blank sample was colorless and transparent to the naked eye. The silica composite with the embedded QDs appeared to be translucent and showed emission of green light from the QDS under UV excitation. The transmission spectra in Fig. 2 show that the blank sample had a transmittance greater than $80 \%$ in
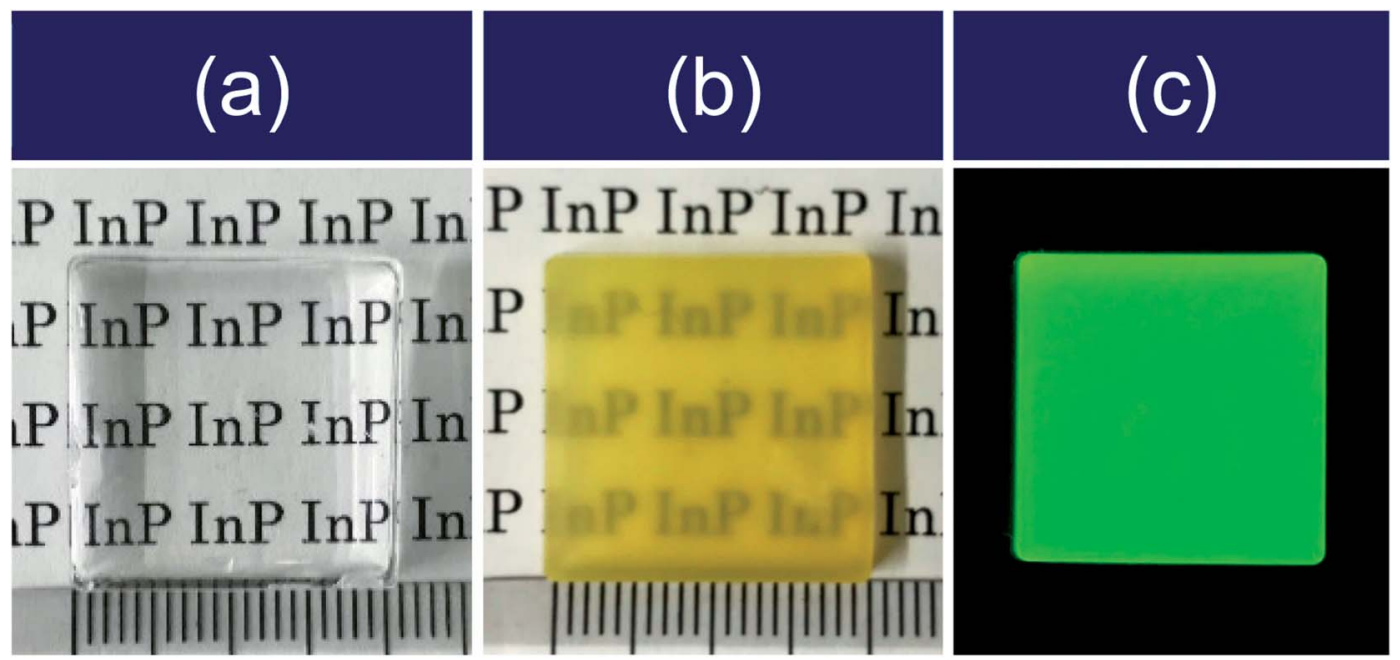

Fig. 1 Photographs of monolithic (a) blank silica and (b and c) silica composite containing InP/ZnS QDs (20 mm $\times 20 \mathrm{~mm} \times 3.6 \mathrm{~mm})$ under (a and b) white light and (c) $365 \mathrm{~nm}$ UV light. 


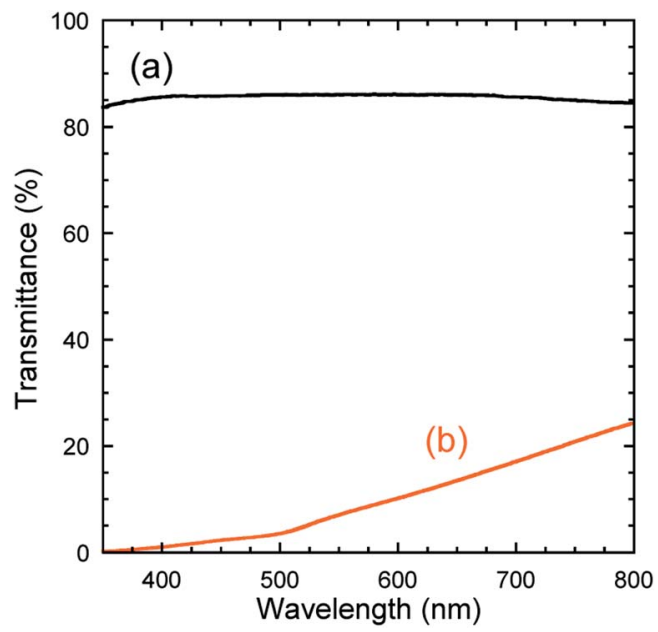

Fig. 2 Transmission spectra of monolithic (a) blank silica and (b) silica composite containing InP/ZnS QDs.

the visible region, whereas the transmittance of the QD-silica composite was less than $25 \%$. This low transmittance was attributed to increased light scattering by QD aggregates. The as-received InP/ZnS QDs modified with DDT were dispersed in TEOS to form a transparent dispersion (see Fig. S2 in ESI $\dagger$ ). The dispersion become turbid within a few minutes of mixing. The QD dispersions modified with DDT therefore did not show longterm stability in TEOS. Furthermore, the hydrophobicity of the sol decreased over time owing to the generation of polar molecules in the sol-gel process, ${ }^{29}$ which promoted QD aggregation.

The normalized PL spectra of the QD dispersions in toluene and TEOS were similar, as shown in Fig. 3. Their color coordinates were outside of the BT.709 color gamut, which is the traditional format of high-definition television, as shown in the chromaticity diagram. However, the PL peak of the monolithic silica composite showed a red-shift, likely because of enhanced self-absorption resulting from QD aggregation. Accordingly, its emission color was closer to the BT.709 color gamut, indicating a loss of green color purity. The PLQY of the monolithic silica composite was $1.6 \%$, which was considerably lower than that of the QD toluene dispersion (67\%). The decrease of PLQY can be attributed to degradation of the QDs in the presence of lactic acid and aggregation effects. The volume fraction of the TEOS : lactic acid used in the fabrication of the monolithic samples was $3: 2$; hence, a large amount of acid remained in the prepared sols. The PLQY decrease should be suppressed by reducing the amount of acid used. We therefore prepared monolithic silica without QDs at a volume fraction of $3: 1$. However, a transparent gel was not obtained under this condition (see Fig. S3 in ESI + ), indicating that this amount of carboxylic acid might not be sufficient to achieve complete and homogeneous gelation of the sol.

\subsection{Characterization of TMOS-modified InP/ZnS QDs}

3.2.1 Structures and compositions. Fig. 4 shows XRD profiles of as-received InP/ZnS QDs, TMOS-modified InP/ZnS QDs $(20 \mathrm{~h})$, and TMOS-modified InP/ZnS QDs $(7 \mathrm{~d})$. These profiles corresponded to the ICDD card data of zinc blende ZnS. The lack of InP peaks was attributed to the low diffraction intensity of InP owing to its strong X-ray absorption. ${ }^{16}$ An amorphous halo of silica was not clearly observed for the TMOSmodified InP/ZnS QDs ( $20 \mathrm{~h}$ and $7 \mathrm{~d}$ ), which suggested that only a small amount of silica coated each QD. However, the XRF analysis confirmed the presence of In and $\mathrm{Si}$ in the TMOSmodified InP/ZnS QDs prepared by both routes. The molar ratio of Si to In for the TMOS-modified InP/ZnS QDs increased from 5.9 to 19.1 when the aging was prolonged from $20 \mathrm{~h}$ to 7 days, which suggested that growth of a silica shell proceeded in this time. FT-IR analysis confirmed the presence of silica, as shown in Fig. 5. In the FT-IR spectrum of TMOS, the peak at
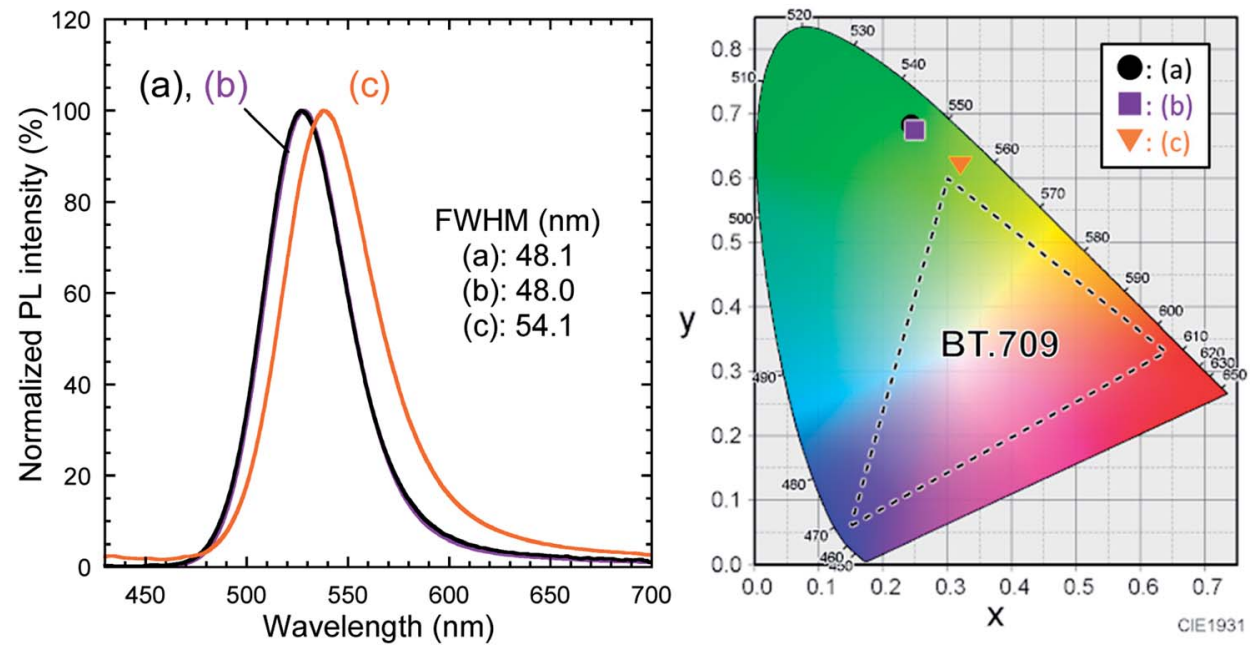

Fig. 3 Normalized PL spectra of InP/ZnS QD dispersions in (a) toluene and (b) TEOS at a concentration of $9.3 \mathrm{mg} \mathrm{mL}^{-1}$, and (c) silica composite containing InP/ZnS QDs. $\lambda_{\mathrm{ex}}=400.0 \mathrm{~nm}$. Their color coordinates are plotted in the CIE 1931 chromaticity diagram (detailed values are summarized in Table $\mathrm{S} 1 \uparrow$ ). 


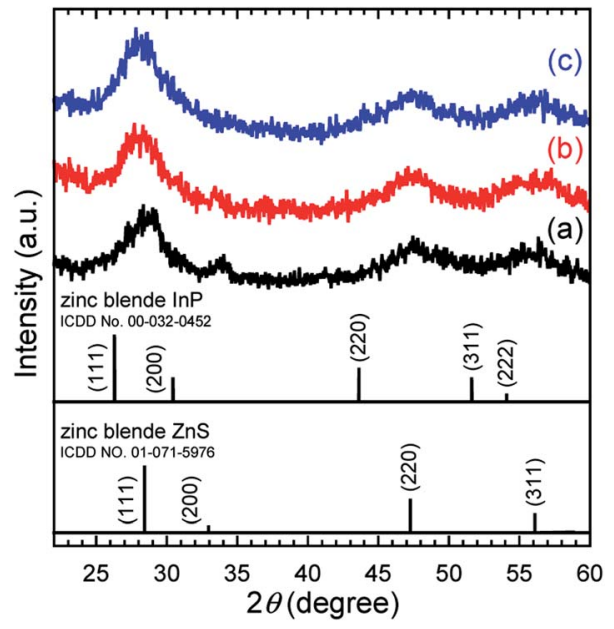

Fig. 4 XRD profiles of (a) as-received InP/ZnS QDs, (b) TMOS-modified InP/ZnS QDs (20 h), and (c) TMOS-modified InP/ZnS QDs (7 d). ICDD card data for zinc blende $\operatorname{InP}$ and $\mathrm{ZnS}$ are also shown for reference.

$\sim 1200 \mathrm{~cm}^{-1}$ was assigned to the symmetric rocking modes of $\mathrm{CH}_{3}(\mathrm{O}) ; \rho_{\mathrm{s}}\left[\mathrm{CH}_{3}(\mathrm{O})\right] .32$ The strong peak at $\sim 1110 \mathrm{~cm}^{-1}$ was attributed to stretching vibrations of $\mathrm{SiO}$ and $\mathrm{CO} ; \nu(\mathrm{SiO})$ and $\nu(\mathrm{CO}){ }^{32}$ The peak appearing at $\sim 840 \mathrm{~cm}^{-1}$ was also assigned to $\nu(\mathrm{SiO})$ and $\nu(\mathrm{CO}) .^{32}$ TMOS was not detected in either of the TMOS-modified QD samples indicating that very little of the TMOS was adsorbed or that the adsorbed TMOS was completely hydrolyzed. Absorption peaks from the deformation modes of $\mathrm{SiOH}$ are located at approximately $840-920 \mathrm{~cm}^{-1}$, depending on the progress of the TMOS hydrolysis. ${ }^{32}$ However, there was no direct evidence for the presence of silanol groups in the measured spectra. A weak peak with a narrow width was observed at $\sim 970 \mathrm{~cm}^{-1}$ for both of the composites derived from the embedded QDs, although a precise assignment could not be determined. The FT-IR spectrum of the TMOS-modified InP/ZnS QDs $(20 \mathrm{~h})$ was similar to that of the as-received InP/ZnS QDs,

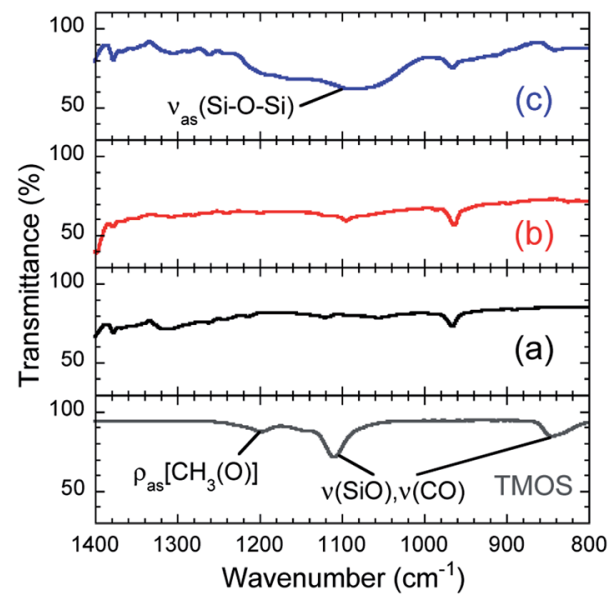

Fig. 5 FT-IR spectra of (a) as-received $\operatorname{InP} / \mathrm{ZnS}$ QDs, (b) TMOSmodified InP/ZnS QDs (20 h), and (c) TMOS-modified InP/ZnS QDs (7 d). TMOS is also shown for reference. although a slight change of the background was observed. Conversely, when the aging duration was prolonged to 7 days, a strong broad band appeared at $\sim 1100 \mathrm{~cm}^{-1}$, assigned to asymmetric stretching vibrations of $\mathrm{Si}-\mathrm{O}-\mathrm{Si} ; \nu_{\text {as }}(\mathrm{Si}-\mathrm{O}-\mathrm{Si}){ }^{33}$ This is a characteristic feature of silica gel. The aging duration of $20 \mathrm{~h}$ was therefore considered to be too short to generate an adequate amount of silica through hydrolysis and condensation of TMOS. From the XRF and FT-IR analyses, we concluded that silica formed in the acid-free solution containing trace amounts of water under stirring at room temperature.

3.2.2 Particle morphologies. TEM images of the as-received InP/ZnS QDs and TMOS-modified InP/ZnS QDs (20 h and $7 \mathrm{~d}$ ) are shown in Fig. 6. The mean particle size of the as-received InP/ZnS QDs was $4.3 \pm 1.2 \mathrm{~nm}$, as calculated from the size distribution by TEM observation [Fig. 6(d)]. An aggregate of QDs, $\sim 30 \mathrm{~nm}$ in size, was observed for the TMOS-modified InP/ ZnS QDs $(20 \mathrm{~h})$. Furthermore, larger aggregates, $60-250 \mathrm{~nm}$ in size, were formed for TMOS-modified InP/ZnS QDs $(20 \mathrm{~h})$. The observed aggregates might be composed of QDs combined with silica derived from TMOS. Prolonged aging promoted silica generation, resulting in the formation of larger aggregates.

3.2.3 Optical properties. Toluene dispersions of InP/ZnS QDs and TMOS-modified InP/ZnS QDs (20 h and $7 \mathrm{~d})$ at a QD concentration of $0.5 \mathrm{mg} \mathrm{mL}^{-1}$ appeared to be highly transparent (see Fig. S4 in ESI $\dagger$ ). Fig. 7 shows the UV-vis absorption spectra and Tauc plots of the dispersions. Herein, the Tauc plots were calculated according to eqn (2) to determine the band gap $\left(E_{\mathrm{g}}\right)$ of the QDs. ${ }^{34}$

$$
(\alpha h v)^{2}=A\left(h v-E_{\mathrm{g}}\right),
$$

where $\alpha$ is the absorbance, $h$ is the Planck constant, $\nu$ is the frequency, and $A$ is a constant. In the measurements of UV-vis absorption spectra, the absorbance values of the solutions at $400.0 \mathrm{~nm}$ were adjusted to be 0.05 by diluting the QD concentration. The three UV-vis absorption spectra overlaid each other over the whole region. The band gap determined from Tauc plots was $2.35 \mathrm{eV}$, revealing that the InP core size remained unchanged through the TMOS modification and aging processes. The InP core diameter was $4.5 \mathrm{~nm}$ as estimated from the band gap based on the effective mass approximation method. ${ }^{35,36}$ This size was close to the mean QD size observed by TEM. The InP core should occupy a large part of the InP/ZnS QD volume; however, its presence was not detected by XRD analysis as discussed above.

Fig. 8 depicts PL spectra of toluene dispersions, for which the absorbance at $400.0 \mathrm{~nm}$ was adjusted to be 0.05 . No changes in the spectral shape and no peak shifts were found, which was consistent with the UV-vis spectroscopy results. As shown in the chromaticity diagram, emission color of the QDs was retained stably. The PLQYs of as-received InP/ZnS QDs and TMOSmodified InP/ZnS QDs $(20 \mathrm{~h})$ dispersed in toluene were $67 \%$ and $62 \%$, respectively. The PLQY of TMOS-modified InP/ZnS QDs $(7 \mathrm{~d})$ was $52 \%$. The TMOS modification and aging process decreased the PLQY; however, the values remained greater than $50 \%$. For control experiments, toluene dispersions of the QDs without TMOS were prepared. After aging for $20 \mathrm{~h}$ or 

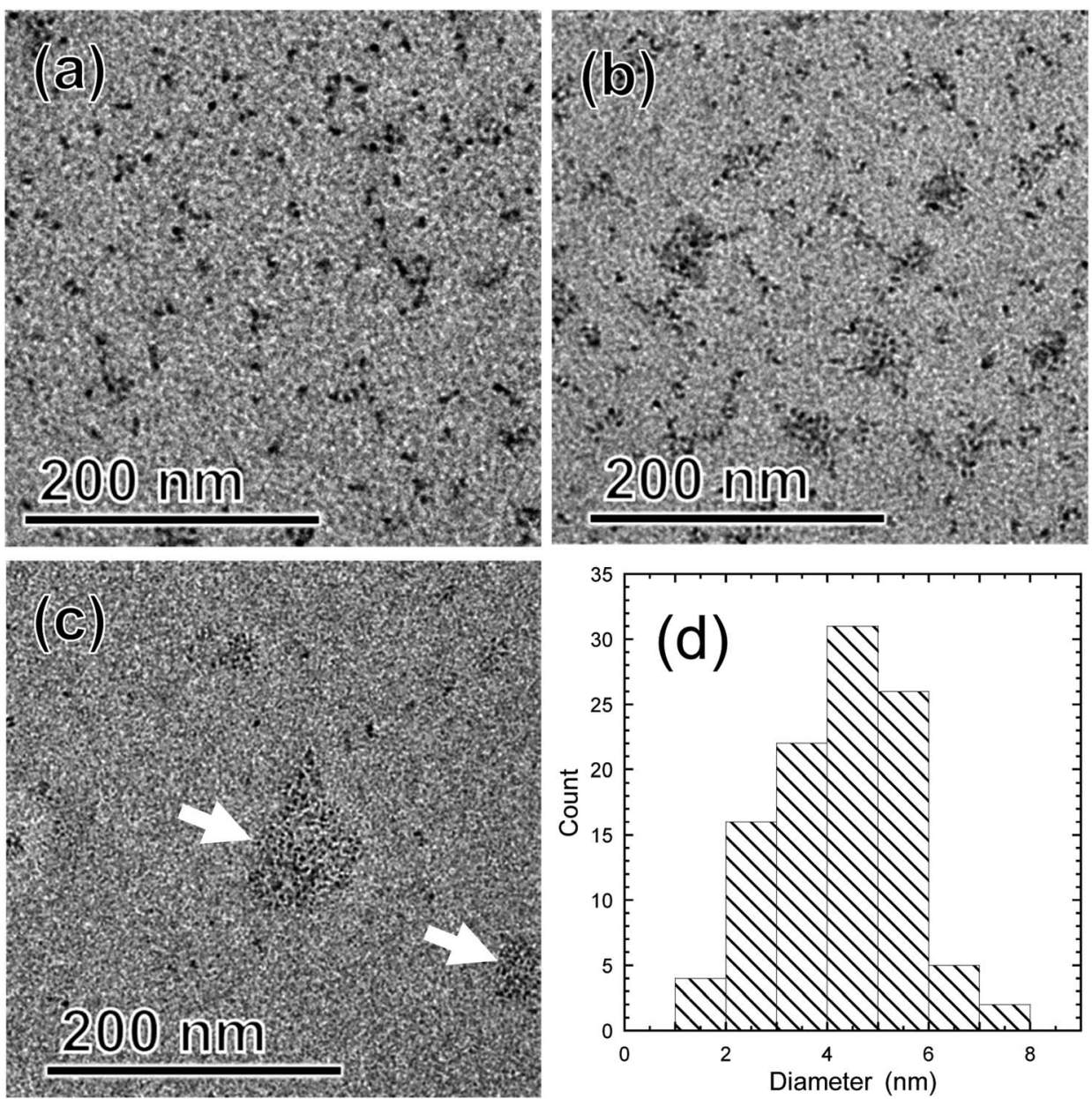

Fig. 6 TEM images of (a) as-received InP/ZnS QDs, (b) TMOS-modified InP/ZnS QDs (20 h), and (c) TMOS-modified InP/ZnS QDs (7 d). (d) Size distribution determined by TEM observations for the as-received InP/ZnS QDs.

7 days, similar decreases of the PLQY were observed in both cases (see Table S2 in ESI $\dagger$ ), indicating that the TMOS modification itself was not responsible for the PLQY decrease. Possible mechanisms for the decrease include desorption of DDT from the QD surface and oxidative degradation of the QD by oxygen dissolved in the toluene during the aging.

Photostability was tested by continuous irradiation with a panel blue LED for $1200 \mathrm{~min}$. PL spectra were recorded over
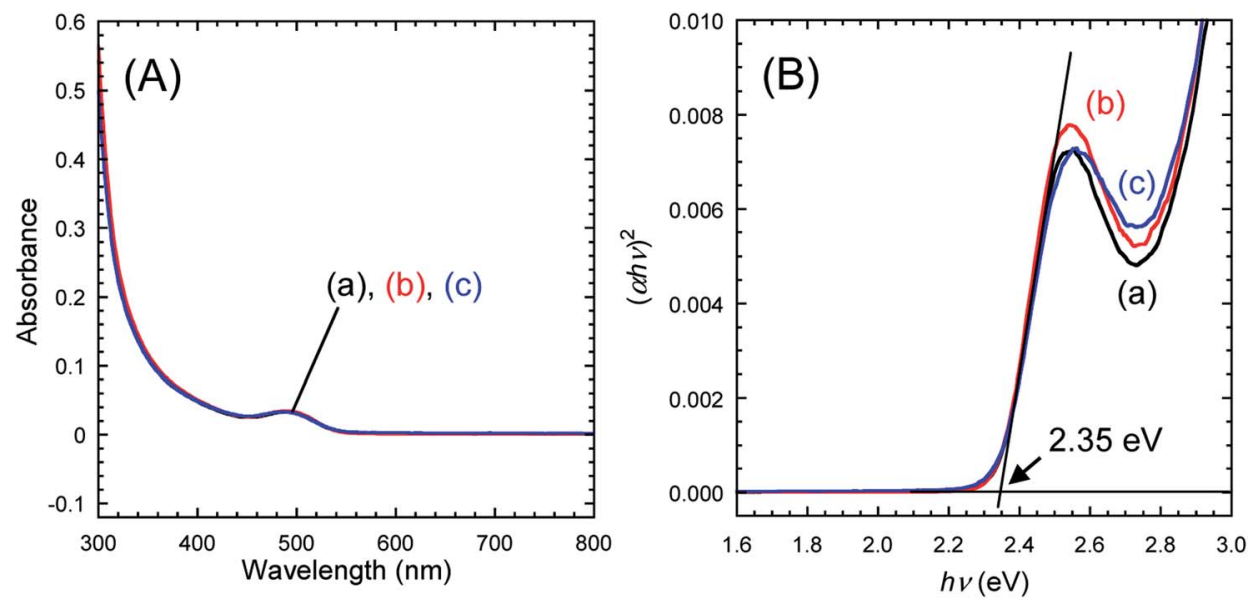

Fig. 7 (A) UV-vis absorption spectra and (B) Tauc plots for toluene dispersions of (a) as-received InP/ZnS QDs, (b) TMOS-modified InP/ZnS QDs (20 h), and (c) TMOS-modified InP/ZnS QDs (7 d). The absorbance of the dispersions at $400 \mathrm{~nm}$ was adjusted to 0.05 by modulating the QD concentration. 

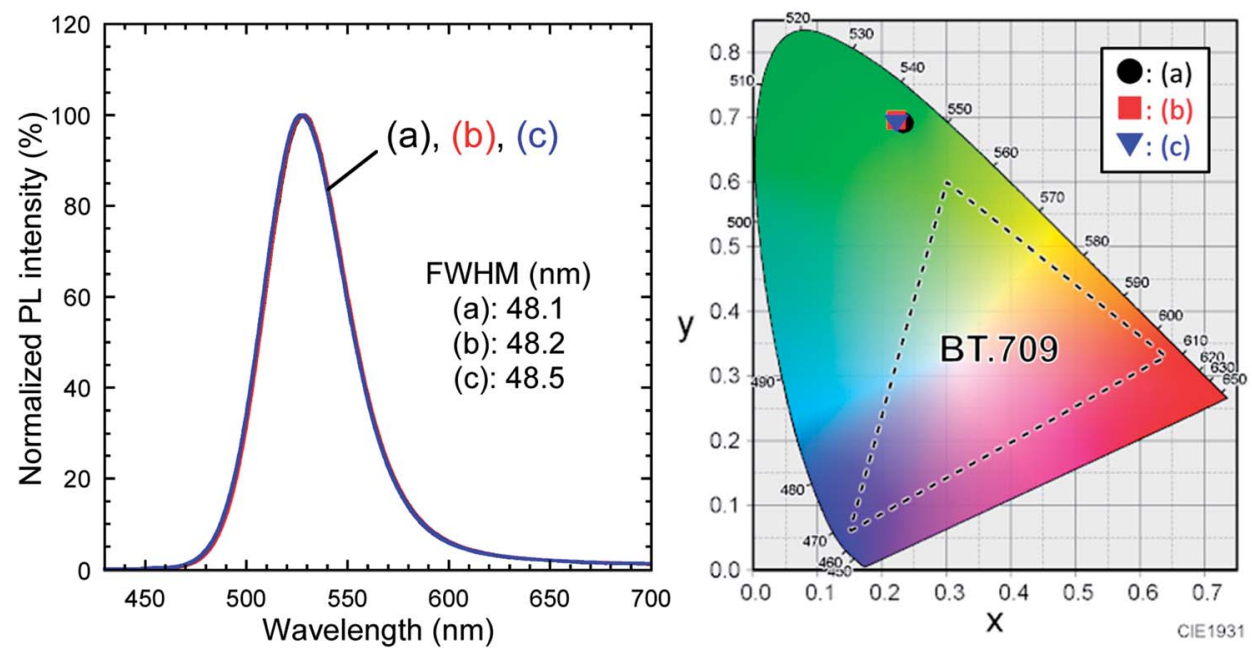

Fig. 8 PL spectra of toluene dispersions of (a) as-received InP/ZnS QDs, (b) TMOS-modified InP/ZnS QDs (20 h), and (c) TMOS-modified InP/ZnS QDs (7 d). $\lambda_{\text {ex }}=400.0 \mathrm{~nm}$. Their color coordinates are plotted in the CIE 1931 chromaticity diagram (detailed values are summarized in Table S1 $\uparrow$ ).

the irradiation duration (see Fig. S5 in ESI $\dagger$ ). The PL peak showed no shift under continuous irradiation for any of the samples. Changes in PL intensity under continuous excitation are plotted in Fig. 9. The PL intensity was normalized at the initial value for each sample. In the early stage of the LED irradiation, we observed an increase of the PL intensity to $125 \%$ of its initial value at $30 \mathrm{~min}$ for the as-received InP/ZnS QDs. This phenomenon has also been reported for thiol-modified InP/ZnS QDs, ${ }^{37}$ although its mechanism has yet to be clarified. Subsequently, the PL intensity decreased to $83 \%$ at $180 \mathrm{~min}$. Conversely, no notable changes were observed for the TMOSmodified InP/ZnS QD samples within $180 \mathrm{~min}$. Hence, the irradiation duration was prolonged to $1200 \mathrm{~min}$. The PL intensity of as-received InP/ZnS QDs finally decreased to $65 \%$. However, the TMOS-modified InP/ZnS QD sample $(20 \mathrm{~h})$ maintained $80 \%$ of its initial value. Furthermore, the final PL

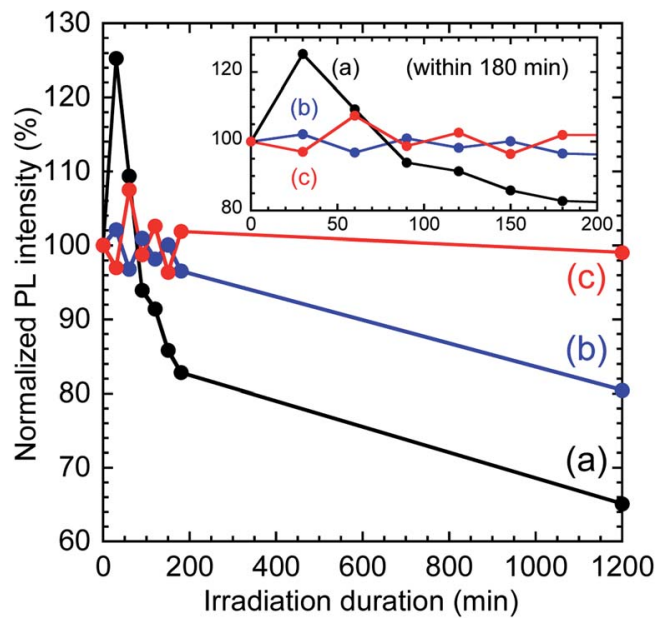

Fig. 9 Changes in PL intensity under continuous irradiation by blue LED. Inset shows the early stage. (a) As-received InP/ZnS QDs, (b) TMOS-modified InP/ZnS QDs (20 h), and (c) TMOS-modified InP/ZnS QDs $(7 \mathrm{~d}) . \lambda_{\mathrm{ex}}=468.3 \mathrm{~nm}$. intensity of the TMOS-modified InP/ZnS QD sample (7 d) was 99\%, indicating its excellent photostability to blue light excitation. These results reveal that the photostability of the InP/ZnS QDs was improved by the silica encapsulation, which protected the QD from photooxidation by oxygen.

The PLQY and photostability of TMOS-modified InP/ZnS QDs (7 d) were superior to those of the TMAS-derived silica composite containing hydrophilized InP/ZnS QDs, as prepared through an aqueous sol-gel method reported in our previous report, ${ }^{16}$ which had a PLQY of $21.7 \%$ and showed PL decrease under blue light irradiation. The final PLQY achieved for the QD-silica composite fabricated by method (ii) was over $50 \%$ demonstrating the excellent photostability.

\section{Conclusions}

In this work, green-emitting InP/ZnS QDs modified with DDT were embedded into silica by two methods to improve their photostability while maintaining a high PLQY and a color purity in green luminescence. Monolithic silica composites from a non-aqueous route based on TEOS and lactic acid showed low transparency, a loss in the green color purity, and a PLQY of $1.6 \%$, owing to QD aggregation in the sol-gel process and degradation of the QDs by the acid. In another method, a toluene dispersion of the QDs added to TMOS was aged for $20 \mathrm{~h}$ or 7 days with stirring at room temperature. The generation of silica was confirmed by FT-IR analysis, which revealed that hydrolysis and condensation of TMOS occurred in the presence of trace water in the system. The PLQY of the TMOS-modified InP/ZnS QDs $(20 \mathrm{~h})$ was $62 \%$, which was slightly lower than the $67 \%$ of the original QDs. The PLQY decreased to $52 \%$ when the aging was prolonged to 7 days. This PLQY decrease was attributed to desorption of surface ligands from the QD surface and oxidative degradation by dissolved oxygen in toluene. Shape and position of the PL peak for each TMOS-modified sample were not changed from those of the as-received QDs. 
The high color purity of green emission therefore was retained well even after silica encapsulation. Photostability testing was performed by continuous irradiation from a blue LED. The decrease of the PL intensity was suppressed by silica encapsulation. In particular, the PL intensity of the TMOS-modified InP/ ZnS QDs (7 d) maintained 99\% of its initial intensity. Silica encapsulation of the InP/ZnS QDs could prevent contact of the QDs with oxygen in the air, resulting in an improvement in the photostability. Consequently, excellent photostability with a PLQY greater than 50\% was achieved for our silica and InP/ ZnS QD composites. The good long-term photostability is attractive for practical applications. In particular, the excellent stability of the highly-pure green PL, in terms of intensity, peak width, and emission wavelength, is desirable for maintaining color tone in wide-color gamut display applications. Highlyphotostable green-emitting fluorescent nanocomposites were successfully obtained through the second route using TMOS preventing significant degradation of PLQY and change in emission color. Such a fluorescent nanomaterial would be applicable to LEDs and bioassay as well as wide-color gamut displays.

\section{Conflicts of interest}

There are no conflicts to declare.

\section{References}

1 C. K. De, T. Routh, D. Roy, S. Mandal and P. K. Mandal, J. Phys. Chem. C, 2018, 122, 964-973.

2 E. Bang, Y. Choi, J. Cho, Y. H. Suh, H. W. Ban, J. S. Son and J. Park, Chem. Mater., 2017, 29, 4236-4243.

3 Y. Wang, Y. Wang, G. Chen, Y. Li, W. Xu and S. Gong, ACS Appl. Mater. Interfaces, 2017, 9, 30297-30305.

4 Y. Z. Wu, J. Sun, Y. Zhang, M. Pu, G. Zhang, N. He and X. Zeng, ACS Appl. Mater. Interfaces, 2017, 9, 13068-13078.

5 S. Xu, J. Ziegler and T. Nann, J. Mater. Chem., 2008, 18, 26532656.

6 X. Yang, D. Zhao, K. S. Leck, S. T. Tan, Y. X. Tang, J. Zhao, H. V. Demir and X. W. Sun, Adv. Mater., 2012, 24, 4180-4185.

7 E. Jang, W. Song, K. Lee and H. Yang, Nanotechnology, 2013, 24, 045607.

8 K. Gugula and M. Bredol, Z. Naturforsch., B: J. Chem. Sci., 2014, 69, 217-223.

9 S. Jun, J. Lee and E. Jang, ACS Nano, 2013, 7, 1472-1477.

10 M. Bouguerra, M. Samah, M. A. Belkhir, A. Chergui, L. Gerbous, G. Nouet, D. Chateigner and R. Madelon, Chem. Phys. Lett., 2006, 425, 77-81.

11 P. Yang, M. K. Lu, C. F. Song, G. J. Zhou, Z. P. Ai, D. Xu, D. R. Yuan and X. F. Cheng, Mater. Sci. Eng., B, 2003, 97, 149-153.

12 M. Wang, Y. Xue, Z. Lin, X. Huo, J. Li and X. Yao, Mater. Lett., 2008, 62, 574-576.

13 N. V. Hullavarad and S. S. Hullavarad, Photonics Nanostructures: Fundam. Appl., 2007, 5, 156-163.
14 S. M. Reda, Acta Mater., 2008, 56, 259-264.

15 P. Yang, M. K. Lu, C. F. Song, M. K. Lu, X. Yin, G. J. Zhou, D. Xu and D. R. Yuan, Chem. Phys. Lett., 2001, 345, 429-434.

16 T. Watanabe, C. Wada, Y. Iso, T. Isobe and H. Sasaki, ECS J. Solid State Sci. Technol., 2017, 6, R75-R80.

17 D. Y. Kim, H. Du, D. W. Johnson Jr and S. Bhandarkar, J. Am. Ceram. Soc., 2004, 87, 1789-1791.

18 Y. Iso, S. Takeshita and T. Isobe, Opt. Mater., 2014, 36, 717722.

19 C. Wada, Y. Iso, T. Isobe and H. Sasaki, RSC Adv., 2017, 7, 7936-7943.

20 S. Kim, T. Kim, M. Kang, S. K. Kwak, T. W. Yoo, L. S. Park, I. Yang, S. Hwang, J. E. Lee, S. K. Kim and S. W. Kim, J. Am. Chem. Soc., 2012, 134, 3804-3809.

21 J. Li, W. Bu, L. Guo, Z. Chen, F. Chen and J. Shi, J. Mater. Res., 2008, 23, 2796-2803.

22 S. Tamang, G. Beaune, I. Texier-Nogues and P. Reiss, ACS Nano, 2011, 5, 9392-9402.

23 H. Kim, M. Suh, B. Kwon, D. S. Jang, S. W. Kim and D. Y. Jeon, J. Colloid Interface Sci., 2011, 363, 703-706.

24 K. Susumu, H. T. Uyeda, I. L. Medintz, T. Pons, J. B. Delehanty and H. Mattoussi, J. Am. Chem. Soc., 2007, 129, 13987-13996.

25 S. Kim and M. G. Bawendi, J. Am. Chem. Soc., 2003, 125, 14652-14653.

26 F. Dubois, B. Mahler, B. Dubertret, E. Doris and C. Mioskowski, J. Am. Chem. Soc., 2007, 129, 482-483.

27 W. Liu, M. Howarth, A. B. Greytak, Y. Zheng, D. G. Nocera, A. Y. Ting and M. G. Bawendi, J. Am. Chem. Soc., 2008, 130, 1274-1284.

28 H. T. Uyeda, I. L. Medintz, J. K. Jaiswal, S. M. Simon and H. Mattoussi, J. Am. Chem. Soc., 2005, 127, 3870-3878.

29 K. G. Sharp, J. Sol-Gel Sci. Technol., 1994, 2, 35-41.

30 S. Huang, Z. Li, L. Kong, N. Zhu, A. Shan and L. Li, J. Am. Chem. Soc., 2016, 138, 5749-5752.

$31 \mathrm{~J}$. Livage and M. Henry, in Ultrastructure Processing of Advanced Ceramics, ed. J. D. Mackenzie and D. R. Ulrich, John Wiley \& Sons, New York, 1988, ch. 12, pp. 183-195.

32 I. S. Ignatyev, M. Montejo, F. Partal Ureña, T. Sundiusc and J. J. López González, Vib. Spectrosc., 2006, 40, 1-9.

33 N. B. Colthup, L. H. Daly and S. E. Wiberley, in Introduction to Infrared and Raman Spectroscopy, Academic Press, New York, 1990, 3rd edn, pp. 361, 414 and 457.

34 J. Tauc and A. J. Menth, J. Non-Cryst. Solids, 1972, 8-10, 569585.

35 M. J. Anc, N. L. Pickett, N. C. Gresty, J. A. Harris and K. C. Mishra, ECS J. Solid State Sci. Technol., 2013, 2, R3071-R3082.

36 X. Y. Wang and N. Herron, Phys. Rev. B: Condens. Matter Mater. Phys., 1990, 42, 7253-7255.

37 C. Yoon, T. Kim, M. H. Shin, Y. G. Song, K. Shin, Y. J. Kim and K. Lee, J. Mater. Chem. C, 2015, 3, 6908-6915. 